\title{
Three-Dimensional Numerical Study of the Acoustic Properities of a Highly Underexpanded Jet
}

\author{
Kun $\mathrm{Wu}^{1}$, Xiaopeng $\mathrm{Li}^{2}$, Wei Yao ${ }^{3}$, Xuejun Fan ${ }^{4}$ \\ State Key Laboratory of High Temperature Gas Dynamics, Institute of Mechanics, Chinese Academy of Sciences, \\ Beijing, 100190, P. R. China
}

The goal of this paper is to advance the simulation of the acoustic field of highly underexpanded jets and to gain a deeper physical understanding of the noise generation mechanism. Large eddy simulation of an underexpanded jet with NPR of 5.60 is implemented with one-equation model for sub-grid scale (SGS) kinetic energy. The predicted time-averaged results agree well with the literature data and theoretical model, especially for the height and diameter of Mach disk. The generation and transport mechanism of sound source are discussed based on high temporal-resolution instantaneous flow fields. Furthermore, the dual-source pattern of the jet noise field is revealed by frequency spectrum analysis.

\section{Nomenclature}

$\begin{array}{ll}p & =\text { static pressure } \\ T & =\text { static tempreture } \\ U & =\text { velocity } \\ \rho & =\text { density } \\ - & =\text { cell average } \\ \sim & =\text { Favre filter } \\ \infty & =\text { parameter of inflow } \\ \tau_{i j} & =\text { viscous stress tensor } \\ h_{s} & =\text { sensible enthalpy per unit mass } \\ q_{i} & =\text { heat flux vector } \\ Y_{k} & =\text { mass fraction } \\ D_{k m} & =\text { equivalent binary mass diffusivity } \\ R & =\text { gas constant of the mixture } \\ R_{u} & =\text { universal gas constant } \\ h_{s} & =\text { enthalpy per unit mass for species } \mathrm{k}\end{array}$

\footnotetext{
${ }^{1} \mathrm{Ph} . D$. Student, State Key Laboratory of High Temperature Gas Dynamics, Institute of Mechanics, Chinese Academy of Sciences, wukun@imech.ac.cn.

${ }^{2}$ Ph.D. Student, State Key Laboratory of High Temperature Gas Dynamics, Institute of Mechanics, Chinese Academy of Sciences, 1xpyfy@163.com.

${ }^{3}$ Associate professor, State Key Laboratory of High Temperature Gas Dynamics, Institute of Mechanics, Chinese Academy of Sciences, yaowei@imech.ac.cn.

${ }^{4}$ Professor, State Key Laboratory of High Temperature Gas Dynamics, Institute of Mechanics, Chinese Academy of Sciences, xfan@imech.ac.cn, Member AIAA.
} 


$\begin{array}{ll}c_{p k} & =\text { specific heat at constant pressure for species } \mathrm{k} \\ \mu_{k} & =\text { dynamic viscosity } \\ S c & =\text { schmidt number } \\ \mathrm{Pr}_{t} & =\text { turbulent Prandtl number } \\ \mathrm{Re} & =\text { Reynolds number } \\ \mathrm{Ma} & =\text { Mach number } \\ \mathrm{M}_{\mathrm{j}} & =\text { perfectly expanded jet Mach number } \\ \mathrm{NPR} & =\text { nozzle pressure ratio } \\ s g s & =\text { sub-grid term } \\ v_{t} & =\text { eddy viscosity } \\ \tilde{S}_{i j} & =\text { strain rate tensor for the resolved scale } \\ C_{v} & =\text { model constants } \\ C_{\varepsilon} & =\text { model constants } \\ \triangle t & =\text { time step } \\ \mathrm{t}_{0} & =\text { integral time scale } \\ \mathrm{D} & =\text { diameter of the nozzle orifice } \\ f_{\mathrm{s}} & =\text { screech frequency } \\ f_{2 \mathrm{~s}} & =\text { second screech frequency }\end{array}$

\section{Introduction}

$\mathrm{H}^{\prime}$

IGHLY underexpanded jets are extensively encountered in various applications including supersonic combustion, aircraft propulsion as well as direct injection gas engines. Especially in scramjet engine, the extremely short residence time of incoming flow in the combustor brings us the challenge that how to obtain a better mixing of the fuel and the main air flow ${ }^{1}$.It is well known that artificially disturbing a flow within a band where the jet is naturally sensitive can effectively enhance mixing. Since the noise is generated by the jet flow field, it is reasonable to expect that the feedback of acoustic field plays an important role in jet excitement and mixing enhancement ${ }^{2}$. However, unlike subsonic jets, which have been extensively studied, the acoustic field generated by highly underexpanded jets is not well understood.

Over the past few decades, significant progress in revealing the source and mechanism of noise generation in underexpanded jets has been made ${ }^{3-7}$. For nearly fifty years, Lighthill's acoustic analogy (1952) is the main theory to explain the aero-acoustic problems. Among the years, many variants of the basic acoustic analogy theory were proposed, but most of them were based on the assumption of quadrupoles sound source ${ }^{8-10}$. In the frame of acoustic analogy theory, the origination and location of sound source are generally not well defined. The characteristic time scale of high speed jets is in the order of $\sim 10^{-6} \mathrm{~s}$, which is far beyond the temporal resolution of traditional

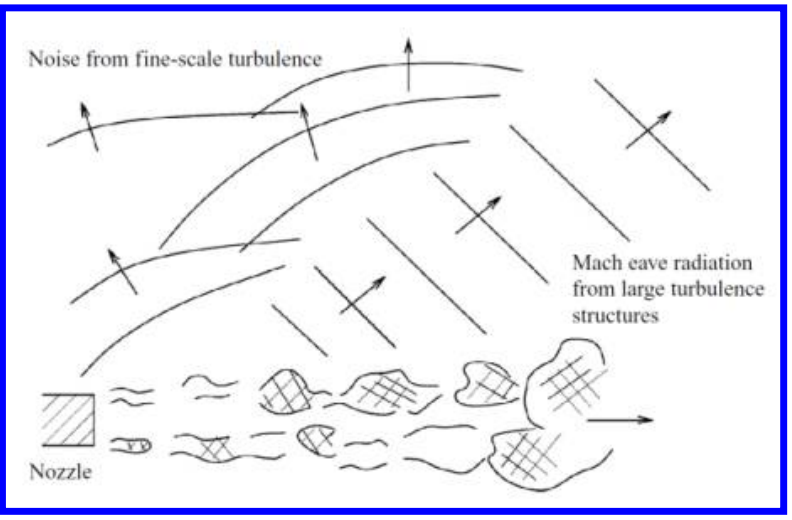

Figure. 1. Schematic of the acoustic field generated by the large-scale turbulent structures and finescale structures feathering directional and none-directional waves in the underexpanded jet flow 
experimental approaches like spark Schlieren photography. Thus numerical modeling with high temporal resolution is adopted to understand the dynamic process of sound source origination and sound wave convection.

In previous studies, Lighthill ${ }^{3}$ analyzed the flow filed of sound production and related the noise production to the fine-scale turbulent eddies, but further studies by Crow and Champagne ${ }^{11}$ and Bradshaw et al. ${ }^{12}$ pointed out that large-scale turbulent structures also play an important role in the sound generation. Figure. 1 taken from Tam ${ }^{13}$ illustrates the concept of two different types of sound sources in the jet flow field, where the two sets of acoustic fields produced by the fine-scale and large-scale turbulent structures respectively have distinguished characteristics. Tam et al (2008) analyzed the source mechanism in high speed jets by four experimental approaches, i.e. singlemicrophone far-field measurements, two-microphone far-field correlation measurements, direct correlation of jet turbulence fluctuations and far-field sound as well as acoustic mirror measurement. However, putting microphone(s) in the flow field will unavoidably change the acoustic characteristics of the jets therefore the measured far-field data can not exactly reveal the sound sources. Besides the measurements using microphone(s) can only provide data at some discrete points which can not give a whole morphology of the acoustic field. Rayleigh-scattering concentrates in a small localized volume within the jet, thus in a nutshell it is also a point measuring approach ${ }^{14}$. Recent years, accurate modeling of aero-acoustic fields is becoming available with the advancement in CFD (Computational Fluid Dynamics) models and computational resources. Numerical modeling can not only give the panorama of the sound field but also the data at points of interest ${ }^{15,16}$. By aid of numerical studies, the acoustic characteristics including screech tone and turbulent mixing noise were discussed but the sound sources and how they were originated and convected downstream remain unclear.

In this study, Large Eddy Simulation (LES) is used to study a highly underexpanded $\mathrm{N}_{2}$ jets. The Reynolds number is on the order of $10^{5}$ with the jet nozzle pressure ratio (NPR) of 5.60.The contents of this paper include: (1) LES modeling is performed based on an in-house developed OpenFOAM ${ }^{17}$ code AstroFoam, with numerical details presented in section II. (2) The numerical results are validated against experimental results and data from literatures. (3) The dynamic process of sound source origination and transportation in the near field of the jets is discussed. (4) The two sound sources in the acoustic field are identified and their emission characteristics are discussed.

\section{Numerical Procedure}

\section{A. Governing Equations}

The compressible flows are governed by the full Navier-Stokes equations. In the LES framework, the filtered equations are:

$$
\begin{gathered}
\frac{\partial \bar{\rho}}{\partial t}+\frac{\partial \bar{\rho} \tilde{u}_{i}}{\partial x_{i}}=0 \\
\frac{\partial \bar{\rho} \tilde{u}_{i}}{\partial t}+\frac{\partial \bar{\rho} \tilde{u}_{i} \tilde{u}_{j}}{\partial x_{j}}=-\frac{\partial \bar{p}}{\partial x_{i}}+\frac{\partial \tilde{\tau}_{i j}}{\partial x_{j}}-\frac{\partial \tau_{i j}^{s g s}}{\partial x_{j}}+\frac{\partial D_{i j}^{s g s}}{\partial x_{j}} \\
\frac{\partial \tilde{\rho} \tilde{h}_{s}}{\partial t}+\frac{\partial \tilde{\rho} \tilde{u}_{j} \tilde{h}_{s}}{\partial x_{j}}=\frac{\partial \bar{p}}{\partial t}+\frac{\partial \tilde{u}_{j} \tilde{t}_{i j}}{\partial x_{i}}-\frac{\partial \bar{q}_{i}}{\partial x_{i}}-\frac{\partial H_{i}^{s g s}}{\partial x_{i}}+\frac{\partial \sigma_{i}^{s g s}}{\partial x_{i}} \\
\frac{\partial \bar{\rho} \tilde{Y}_{k}}{\partial t}+\frac{\partial \bar{\rho} \tilde{u}_{j} \tilde{Y}_{k}}{\partial x_{j}}=\frac{\partial}{\partial x_{j}}\left(\bar{\rho} D_{k m} \frac{\partial \tilde{Y}_{k}}{\partial x_{j}}\right)-\frac{\partial \Phi_{k, j}^{s g s}}{\partial x_{j}}-\frac{\partial \theta_{k, j}^{s g s}}{\partial x_{j}} \\
\bar{p}=\bar{\rho} R \tilde{T}+R_{u} \sum_{k=1}^{N} T^{s g s}
\end{gathered}
$$

In the above equations, “ " means Favre filter, “-” means cell average, $\rho$ is density, $u_{i}$ is the velocity in $x_{i}$ direction, $p$ is the pressure, $\tau_{i j}$ is the viscous stress tensor, $h_{s}$ is the sensible enthalpy per unit mass, $q_{i}$ is the heat flux vector, 
$Y_{k}$ is the mass fraction, $D_{k m}$ is the equivalent binary mass diffusivity, $T$ is the temperature, $R$ is the gas constant of the mixture gas, $R_{u}$ is the universal gas constant.

The thermodynamic and transport characteristics of individual species, such as the enthalpy per unit mass $h_{k}$ and the specific heat at constant pressure $c_{p k}$, are calculated based on NIST-JANAF thermo-physical and transport database ${ }^{18}$. The dynamic viscosity $\mu_{k}$ is computed by Sutherland's law. The Schmidt number $S c$ and the turbulent Prandtl number $\mathrm{Pr}_{t}$ in the species concentration and energy equations are assumed to be constant of 1.0.

This set of equations are solved using an in-house developed supersonic compressible solver AstroFoam. The second-order semi-discrete, non-staggered KT (Kurganov and Tadmor) central-upwind scheme ${ }^{19}$ is used to solve the advection-diffusion equation. A TVD (Total Variation Diminishing) scheme ${ }^{20}$ with minmod limiter is applied to reconstruct the primitive values at faces to obtain second order accuracy. Time integration is advanced by a secondorder Crank-Nicolson scheme.

\section{B. LES Sub Grid Scale (SGS) Model}

In LES the SGS closure models are needed to model the term superscript as $s g s$ in Equation (1) (5). Some sub grid scales terms as $D_{i j}^{s g s}, \sigma_{i}^{s g s} \theta_{k, j}^{s g s}$ are reported to be small and generally neglected in the previous studies ${ }^{21-23}$. However, the last three unclosed terms $H_{i}^{s g s}, \Phi_{k, j}^{s g s}, \tau_{i j}^{s g s}$ are modeled based on the eddy viscosity assumption as followed:

$$
\begin{gathered}
H_{j}^{s g s}=-\bar{\rho} \frac{v_{t}}{\operatorname{Pr}_{t}} \frac{\partial \tilde{H}}{\partial x_{j}}=-\bar{\rho} \frac{v_{t}}{\operatorname{Pr}_{t}}\left(\frac{\partial \tilde{h}}{\partial x_{j}}+\tilde{u}_{i} \frac{\partial \tilde{u}_{i}}{\partial x_{j}}+\frac{\partial k^{s g s}}{\partial x_{j}}\right) \\
\Phi_{k, i}^{s g s}=-\bar{\rho} \frac{v_{t}}{S c_{t}} \frac{\partial \tilde{Y}_{k}}{\partial x_{i}} \\
\tau_{i j}^{s g s}-\frac{2 \delta_{i j}}{3} \bar{\rho} k^{s g s}=-2 v_{t} \bar{\rho}\left[\tilde{S}_{i j}-\frac{\delta_{i j}}{3} \tilde{S}_{k k}\right]
\end{gathered}
$$

where $v_{t}$ is the eddy viscosity and will be discussed in detail next. $\tilde{S}_{i j}$ is the strain rate tensor for the resolved scale and is defined by:

$$
\tilde{S}_{i j}=\frac{1}{2}\left(\frac{\partial \tilde{u}_{i}}{\partial x_{j}}+\frac{\partial \tilde{u}_{j}}{\partial x_{i}}\right)
$$

In order to determine the eddy viscosity $v_{t}$, a one-equation $\operatorname{model}^{24}$, in which a transport equation for the subgrid turbulent kinetic energy $k^{s g s}$ is provided to account for the non-equilibrium effects, is applied in the current work, and the corresponding expression is:

$$
\begin{gathered}
\frac{\partial \bar{\rho} k^{s g s}}{\partial t}+\frac{\partial \bar{\rho} \tilde{u}_{j} k^{s g s}}{\partial x_{j}}=\frac{\partial}{\partial x_{j}}\left[\bar{\rho}\left(\frac{v_{t}}{\operatorname{Pr}_{t}}+v\right) \frac{\partial k^{s g s}}{\partial x_{j}}\right]-\tau_{i j}^{s g s} \frac{\partial \tilde{u}_{i}}{\partial x_{j}}-C_{\varepsilon} \frac{\bar{\rho}\left(k^{s g s}\right)^{3 / 2}}{\bar{\Delta}} \\
v_{t}=C_{v} \bar{\Delta} \sqrt{k^{s g s}}
\end{gathered}
$$

where $C_{v}$ and $C_{\varepsilon}$ are model constants, and are set to be 0.67 and 1.0.

\section{Numerical Setup}

The computational domain employed for the LES of highly underexpanded sonic jets in the present study is depicted in figure.2. The computational domain mainly consists of a box of size $50 \times 100 \times 50 \mathrm{~mm}$ respectively in $\mathrm{x}, \mathrm{y}$, and $\mathrm{z}$ directions. In order to capture the acoustic feedback phenomenon correctly, a convergent nozzle geometry is 
included in the upstream part of the computational domain. Pure nitrogen with total pressure $\mathrm{P}_{0}$ and total temperature $T_{0}$ is injected into the quiescent air (with stastic pressure $P_{\infty}$, and stastic temperature $T_{\infty}$ ) from a contoured round nozzle of $2.0 \mathrm{~mm}$ in diameter. Figure 2 (c) shows the three-dimensional schematic of the nozzle.

In the present work, the quiescent air in the box-like computational domain is the mixture of nitrogen 0.76699 and oxygen 0.23301 by weight, and the temperature, pressure, density, and velocity are respectively uniform, $\mathrm{T}_{\infty}$ $=300 \mathrm{~K}, \mathrm{P}_{\infty}=101325 \mathrm{~Pa}$, . The simulations is carried out for NPR=5.60, which is a typically highly underexpanded jets. The flow velocities at the nozzle exit is considered to be sonic. The Reynolds number at the nozzle exit is about $\operatorname{Re} \sim 10^{5}$. The details of the simulation conditions are presented in table 1 .

As for the boundary condition, a total pressure and temperature is employed at the nozzle inlet. A zero-gradient condition for velocity is used for the inflow boundary. All walls including the sides of nozzle and the round tube outside the nozzle are treated as no-slip adiabatic walls. At the top of the computational domain together with the four free surfaces, a far field boundary condition is applied.

The integral time scale can be defined using the nozzle diameter $\mathrm{D}$ and the maximum velocities in the near field of the jets as $\mathrm{t}_{0}=\mathrm{D} / 2 \mathrm{U}_{1} \approx 2.5 \times 10^{-6} \mathrm{~s}$. The flow in the nozzle is subsonic and the nozzle exit pressure $\mathrm{P}_{1}$ builds up solely due to the nozzle flow itself. The flow in the nozzle reached a quasi-steady-state as indicated by the pressure profiles around at time $t \approx 80 t_{0}$, when the nozzle exit pressure $P_{1}$ is about $0.3 \mathrm{MPa}$ for NPR=5.60 and the mass flow approximate to constant as well. The flow-through time for the jets washing out the computational domain is about $200 t_{0}$, and thus the total simulation duration is set as $800 t_{0}$ to ensure long enough quasi-steady period for turbulence statistics. The instantaneous results are saved every $2 \mathrm{t}_{0}$, and then turbulence statistics are collected for the last three flow-through times (200t $\mathrm{t}_{0} \sim 800 \mathrm{t}_{0}$, total 300 time steps).

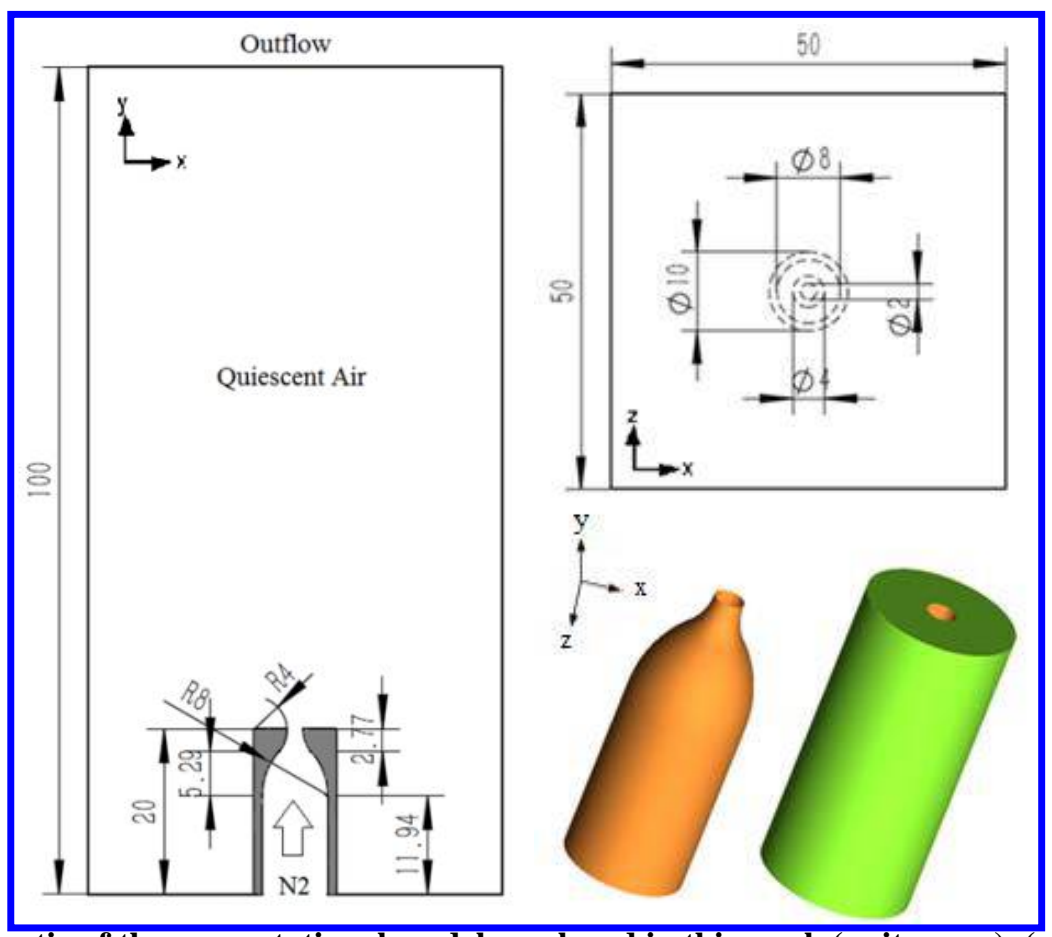

Figure. 2. Schematic of the computational model employed in this work (units: $\mathrm{mm}$ ). (a) Left: the cross streamwise view, (b) Right top: top view, (c) Right bottom: three-dimensional schematic of the nozzle 
Table 1. Flow parameters and simulation conditions.

\begin{tabular}{llcc}
\hline \hline Property & Symbol & Case & Units \\
\hline Mach number at nozzle exit & $\mathrm{M}_{1}$ & 1.0 & - \\
Static pressure at nozzle exit & $\mathrm{P}_{1}$ & 0.3 & $\mathrm{MPa}$ \\
Stagnation pressure & $\mathrm{P}_{0}$ & 0.57 & $\mathrm{MPa}$ \\
Static temperature at nozzle exit & $\mathrm{T}_{1}$ & 300.0 & $\mathrm{~K}$ \\
Density at nozzle exit & $\rho_{1}$ & 3.37 & $\mathrm{~kg} / \mathrm{m}^{3}$ \\
Velocity at nozzle exit & $\mathrm{U}_{1}$ & 353.1 & $\mathrm{~m} / \mathrm{s}$ \\
Reynolds number at nozzle exit & $\mathrm{Re}_{1}$ & 1.36 & $\times 10^{5}$ \\
Static pressure ratio & $\mathrm{P}_{1} / \mathrm{P}_{\infty}$ & 2.96 & - \\
Nozzle pressure ratio (NPR) & $\mathrm{P}_{0} / \mathrm{P}_{\infty}$ & 5.60 & - \\
\hline
\end{tabular}

\section{Grid Generation}

The hexahedral, block-structured grid employed in the present study is refined in the regions of the jet core and the jet shear layers to achieve a high spatial resolution. The overall mesh consists of $27.3 \mathrm{M}$ computational cells, on which the subsequent results are based.

The cells near the edge of the jet orifice have the size of $0.01 \mathrm{~mm}$ the mesh. A refinement region is added to cover the jet core and shear layer which is of great interest in the present study. The cell size increases to $0.1 \mathrm{~mm}$ between the span of $\mathrm{z}=0 \sim 5 \mathrm{~mm}$ and reaches $0.2 \mathrm{~mm}$ at $\mathrm{z}=10 \mathrm{~mm}$. The cell size then increases to $1.0 \mathrm{~mm}$ at the edge of the computational domain. The mesh in the streamwise direction is extruded upwards with an initial cell size of 0.03 $\mathrm{mm}$ and a maximum of $0.08 \mathrm{~mm}$ at $10 \mathrm{~mm}$ height from the nozzle exit. Then the mesh is stretched to a maximum cell size of $0.3 \mathrm{~mm}$ at $70 \mathrm{~mm}$ from the nozzle exit, and a maximum cell size of $0.5 \mathrm{~mm}$ is introduced at the computational exit to ensure computational stability. In the central part of the nozzle, the cell size is $0.024 \mathrm{~mm}$ in the transverse direction and then decreases to $0.01 \mathrm{~mm}$ at the edge of the jet orifice. The cell size of the nozzle in the streamwise is $0.3 \mathrm{~mm}$, and the resolution starts to increases at $\mathrm{y}=3 \mathrm{~mm}$ from the nozzle exit to a maximum cell size of $0.04 \mathrm{~mm}$ at the nozzle exit. In Table 2 the grid resolutions in the near field of the present work are compared with those used in previous LES modeling of supersonic jets ${ }^{25-28}$.

\begin{tabular}{|c|c|c|c|c|c|c|c|}
\hline Cell & $\mathbf{D}(\mathbf{m m})$ & $\Delta \mathbf{r}_{\min }$ & $\Delta \mathbf{r}_{\max }$ & $\Delta \mathbf{y}_{\min }$ & $\Delta \mathbf{y}_{\text {max }}$ & $\operatorname{Re}$ & Total $\left(\times 10^{6}\right)$ \\
\hline Present & 2.0 & $\mathrm{D} / 200$ & $\mathrm{D} / 52$ & $\mathrm{D} / 67$ & $\mathrm{D} / 25$ & $\sim 10^{5}$ & 27.3 \\
\hline Gorle et $a^{25}$ & 2.0 & $\mathrm{D} / 100$ & $\mathrm{D} / 50$ & $\mathrm{D} / 100$ & $\mathrm{D} / 25$ & $\sim 10^{5}$ & 17.4 \\
\hline Vuorinen et $a l^{26}$ & 1.4 & $\mathrm{D} / 70$ & $\mathrm{D} / 50$ & $\mathrm{D} / 35$ & $\mathrm{D} / 25$ & $\sim 10^{5}$ & 12.0 \\
\hline Dauptain $e t a l^{27}$ & 25.4 & $\mathrm{D} / 35$ & $\mathrm{D} / 30$ & $\mathrm{D} / 35$ & $\mathrm{D} / 30$ & $\sim 10^{6}$ & 22.0 \\
\hline Rana et $a^{28}$ & 4.0 & $\mathrm{D} / 33$ & $\mathrm{D} / 33$ & $\mathrm{D} / 33$ & $\mathrm{D} / 33$ & $\sim 10^{4}$ & 9.2 \\
\hline
\end{tabular}

The computational time step is approximately $\Delta \mathrm{t} \approx 1.37 \times 10^{-8} \mathrm{~s}\left(\Delta \mathrm{t} \cdot a / \mathrm{D}=2.42 \times 10^{-3}, a\right.$ is the speed of sound at the nozzle exit), thereby the maximum Courant-Friedrichs-Lewy (CFL) number is 0.6 .

\section{A. Mean Flow and shock spacing}

\section{Result Discussion}

First, the time-averaged contours of axial velocity, density gradient, Mach number and pressure are presented in figure 3(a-d) respectively. The highly underexpanded jet undergoes an initial expansion once bursts into the quiescent air, then due to the pressure imbalance a train of alternating shock cell emerges. Through the first normal shock which is called Mach disk, the flow bears violent variation in static temperature and pressure. Beyond the 
Mach disk, the reflected shock and the jet shear layer intersect with each other to form the triple point. The parallel slip lines start from the triple point and persistent until the shear layer is ended by the flow instability. The modeling results are then compared with available Schlieren image ${ }^{29}$, which indicates that the main characteristics of the highly underexpanded jet, such as the Mach disk, the triple point and Mach barrel, are all well captured.

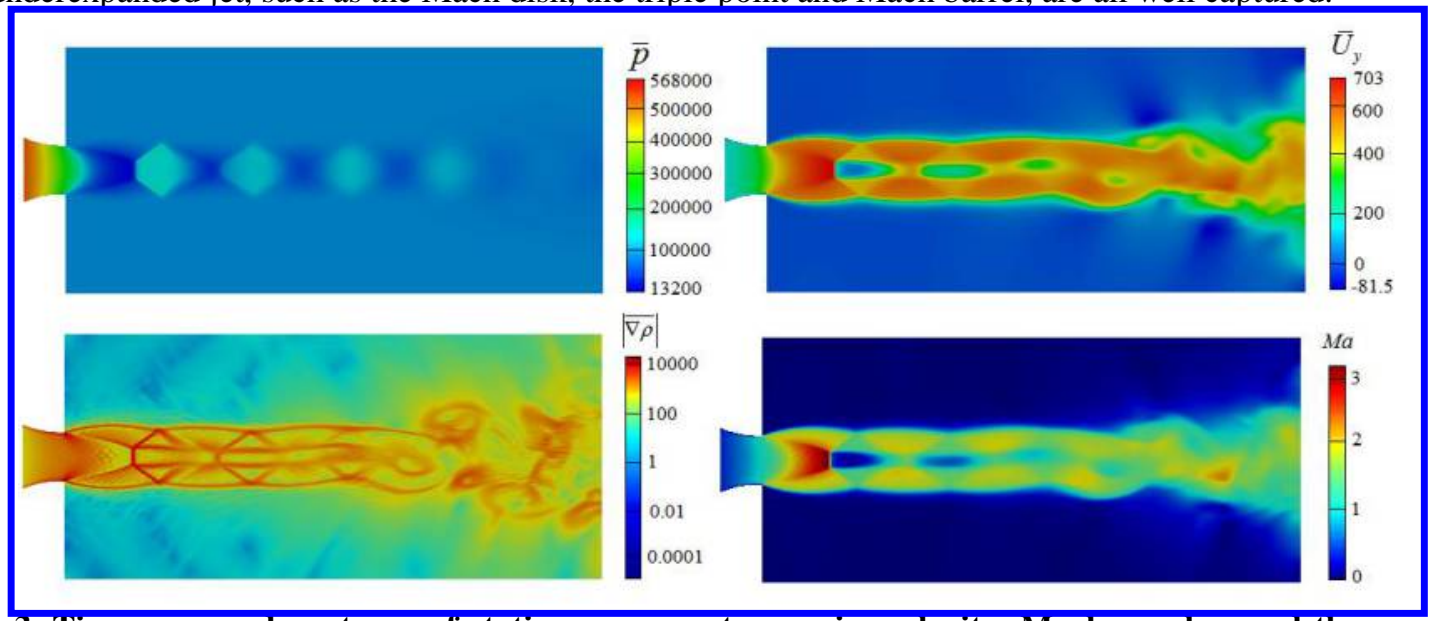

Figure 3. Time-averaged contours of static pressure, streamwise velocity, Mach number and the magnitude of density gradient

In figure 4 (a) the first one shock is used to measure the height and diameter of Mach disk since it is the most important characteristic of the highly underexpanded jet. Ashkenas and Sherman ${ }^{30}$ obtained an empirical formula for the Mach disk height $H_{m}$ for the range of NPR from 20 to 200 as:

$$
\frac{H_{m}}{D}=C_{H} \cdot \sqrt{\frac{P_{0}}{P_{\infty}}}
$$

where $C_{H}$ is a constant of 0.67 . Ewan and Moodie ${ }^{31}$ proposed a much smaller value of $C_{H} \approx 0.55$ for NPR $<10$ but with $C_{H}$ approaching 0.67 with increasing NPR. With the present LES results, $C_{H}$ is considered to be of 0.60, which is between the experimental measurements by Ewan and Moodie and Ashkenas and Sherman yet close to the LES prediction by Vuorinen $e t \mathrm{al}^{26}$, which is shown in table 3.
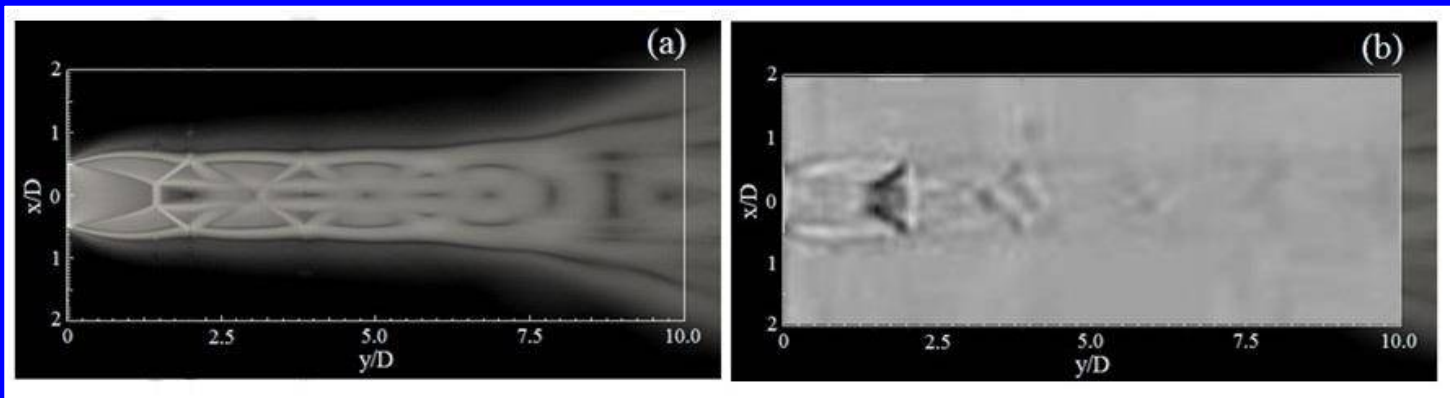

Figure 4. Comparison of the wave structures of present LES and Schlieren graph

Table 3. Comparison of the Mach disk height and diameter

\begin{tabular}{lcccccc}
\hline \hline Author & Technique & $\mathrm{D}(\mathrm{mm})$ & $\mathrm{P}_{\mathrm{o}} / \mathrm{P}_{\infty}$ & $\mathrm{H}_{\mathrm{m}} / \mathrm{D}$ & $\mathrm{W}_{\mathrm{m}} / \mathrm{D}$ & $C_{H}$ \\
\hline Ashkenas and Sherman & $\operatorname{Exp}$ & - & $20 \sim 200$ & - & - & 0.67 \\
\hline Ewan and Moodie & Exp & - & $\sim 10$ & - & - & 0.55 \\
\hline Vuorinen et al & LES & 1.4 & 5.50 & 1.43 & 0.36 & 0.61 \\
\hline Present work & LES & 2.0 & 5.60 & 1.43 & 0.38 & 0.60 \\
\hline \hline
\end{tabular}




\section{B. Sound Source Visualization}

Schlieren photography has been extensively used in the analysis of mean flow characteristics for highly underexpanded jets, however, suffered from its low temporal resolution, the instantaneous characteristics is still not well experimentally revealed. For this reason, modeling results with high temporal resolution are used to analyze the dynamic process of the sound source origination and convection to the downstream.

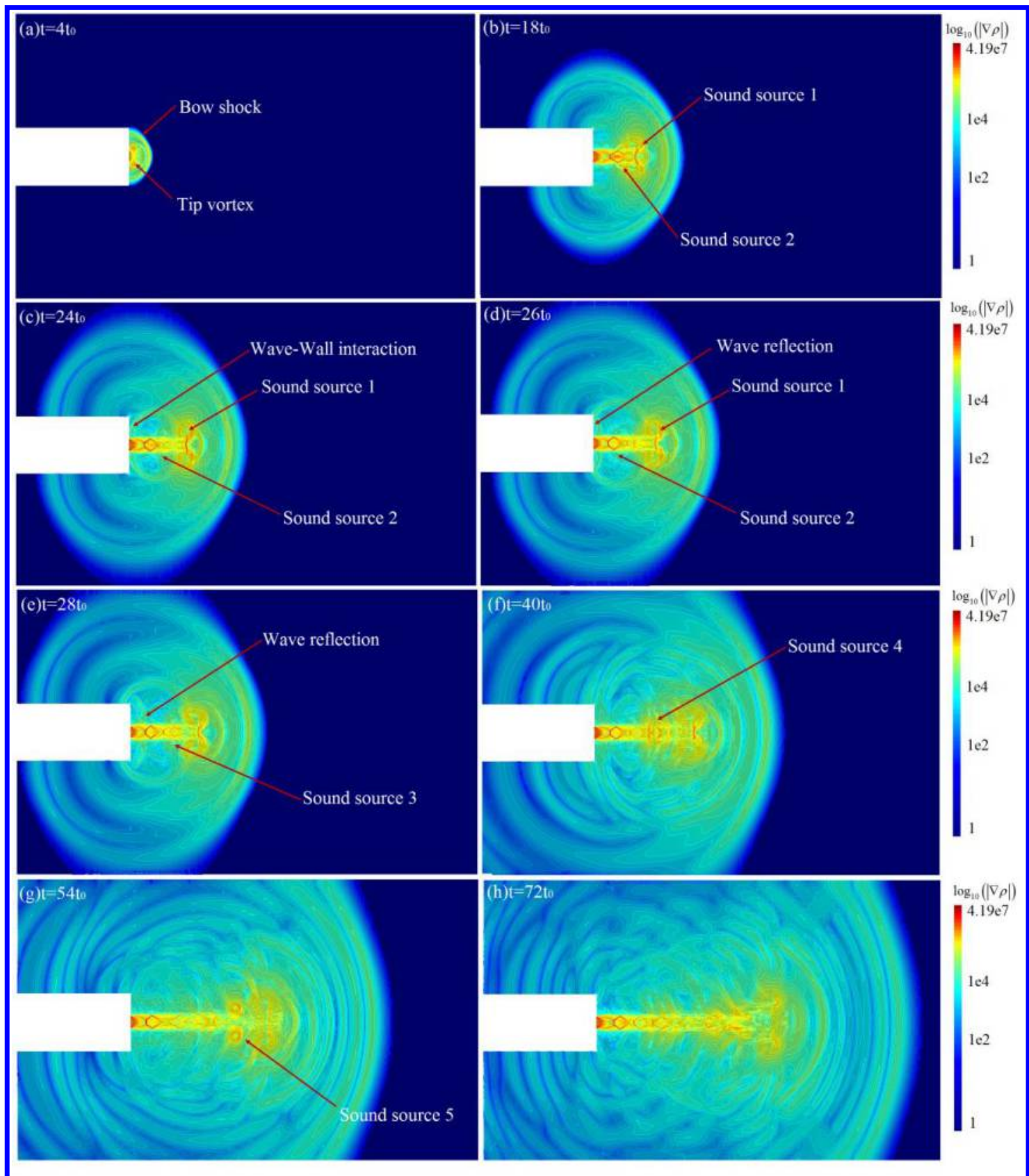

Figure 5. Instantaneous density gradient: showing sound sources origination and convection along with wave's propagation in the flow field (a) $t=4 t_{0}$ (b) $t=18 t_{0}$ (a) $t=24 t_{0}$ (a) $t=26 t_{0}$

Figure 5 shows the typical contours of instantaneous pressure gradient and the flow along with the acoustic field. In figure 5 (a), a bow shock accompanied by a vortex ring emerges as the jet bursts into quiescent air. Then the tip vortex named SS1 (sound source 1) acts as an intensive sound source to radiate sound wave and a new sound source SS2 is generated around the second normal shock wave. Figure.5(c) (d) show the convection of SS1 and SS2 in the 
streamwise direction. The whole interaction process of the sound wave with the nozzle lip and the subsequent reflection into the opposite direction can be observed from the high-temporal-resolution and high-spatial-resolution contours.

In figure $5(\mathrm{~d})$, the reflected sound wave and the counter-streamwise propagating one impact with each other near the Mach disk. At the same time, a new sound source SS3 emerges in the middle of the third Mach barrel. At y 3.5D in the streamwise direction, a new sound source SS4 emits squamiform waves rather than circumferential sound waves. In figure 5 (e) as the jet core grows longer, the deformation of jet shear layer due to Kelvin-Helmholtz instability leads to a new intensive sound source SS5. Figure $5(\mathrm{~g})$ presents a statistically-steady state of the whole jet acoustic field, where some of the initial sound sources disappeared or merged with each other and three intensive sound wave emission regions, i.e. $y \sim 4 \mathrm{D}, 8 \mathrm{D}, 12 \mathrm{D}$ in the streamwise direction, can be observed.

\section{Dual Sound Source Pattern}

Figure 6 presents a panorama of the instantaneous acoustic field of the highly underexpanded jet. In the flow field, due to the intensive turbulent fluctuation, the jet core loses its stability and some large scale coherent structures wrap around it. Because of the existence of wavy jet shear layer, the parallel directional wave named K-H wave (KelvinHelmholtz wave) emits from the jet core at almost the same Mach angle given in Tam ${ }^{32}$. The large eddy turbulent structures are considered to be the source of the highly directional waves in the streamwise direction. Additionally, the interaction of small scale turbulent structures produces lower-intensity broadband waves that prorogating in all directions. Those two wave patterns interact with each other and the dominant wave amplitude oscillates between $\mathrm{K}$ $\mathrm{H}$ wave and sound wave. Similar dual-source pattern of the jet noise field has also been experimentally observed by $\mathrm{Tam}^{32}$.

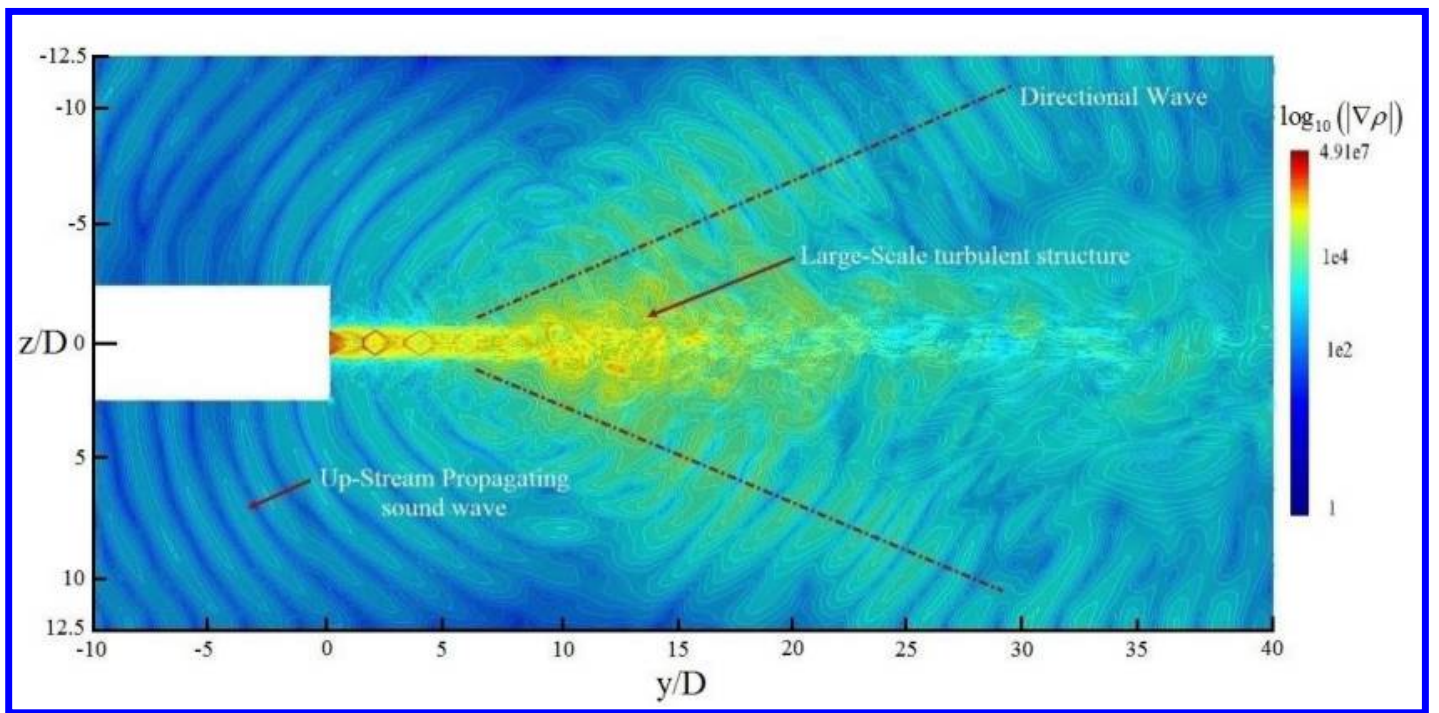

Figure 6. Instantaneous density gradient: showing the two distinct sound wave patterns in the acoustic field including the up-stream propagating none-directional sound waves and the directional waves in the downstream direction.

In the LES modeling, an array of pressure probes are laid in the flow field to analyze the dual-source pattern of the jet noise field, by using the pressure fluctuation historical data at 10 locations, i.e. $y / D=2,4,6,8,10,15,20,25$, 30 and 35 along the lines. Figure 7 presents the FFT (Fast Fourier Transformation) spectrum of the pressure fluctuation data probed at $x / D=10$ and $y / D=8$, where the weak broadband turbulent mixing noise and intensive discrete screech tones are observed. Two additional screech tones $\mathrm{f}_{\mathrm{s}}=37.086 \mathrm{~Hz}$ and $\mathrm{f}_{2 \mathrm{~s}}=45.695 \mathrm{~Hz}$ identical to those observed by Panda ${ }^{33}$ are also shown in the power spectrum. The FFT spectra of pressure fluctuations at the other 9 
locations are similar with that at $x / D=10$ and $y / D=8$. The screech tones are identical for the 10 locations except the amplitude, i.e. the intensity of the acoustic field at different locations differs.

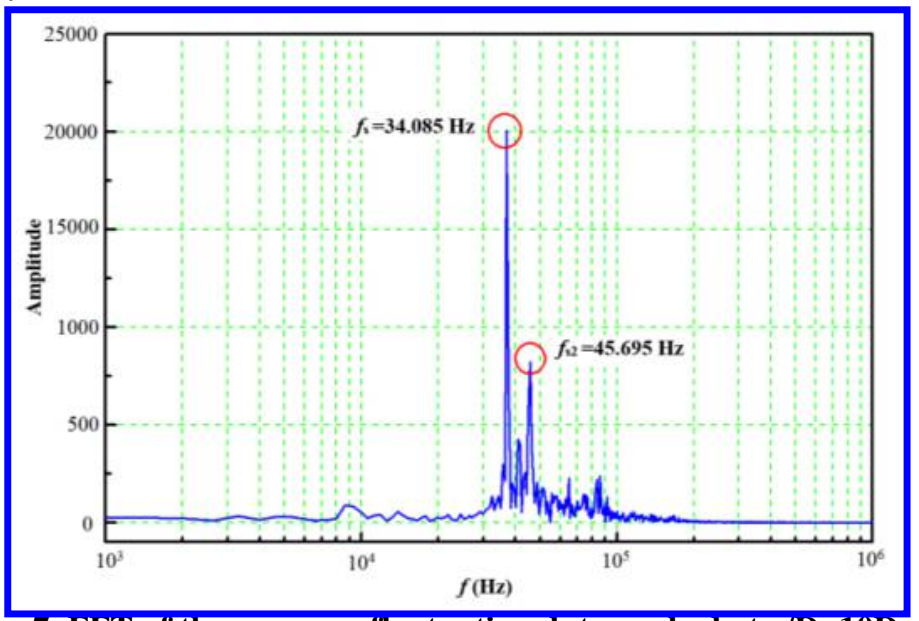

Figure 7. FFT of the pressure fluctuation data probed at $x / D=10 \mathrm{D}$ and $\mathrm{y} / \mathrm{D}=8$

The dual-source pattern can be revealed through analysis on the intensity of the acoustic field at various locations. Figure 8 compares the amplitude of the screech tone at different locations. It can be seen that in the region of $y / D>15$, the acoustic intensity increase rapidly due to the superposition of K-H wave and sound wave. However, the directional K-H wave are weak for quasi-steady jet plume in the near-field region of $y / D<10$, where the intensity of acoustic field is mainly determined by the sound wave. The amplitude of the acoustic field in those two regions varies significantly, suggesting the existence of two types of sound sources in the highly underexpanded jet, which is consistent with the experimental observation of Panda ${ }^{33}$.

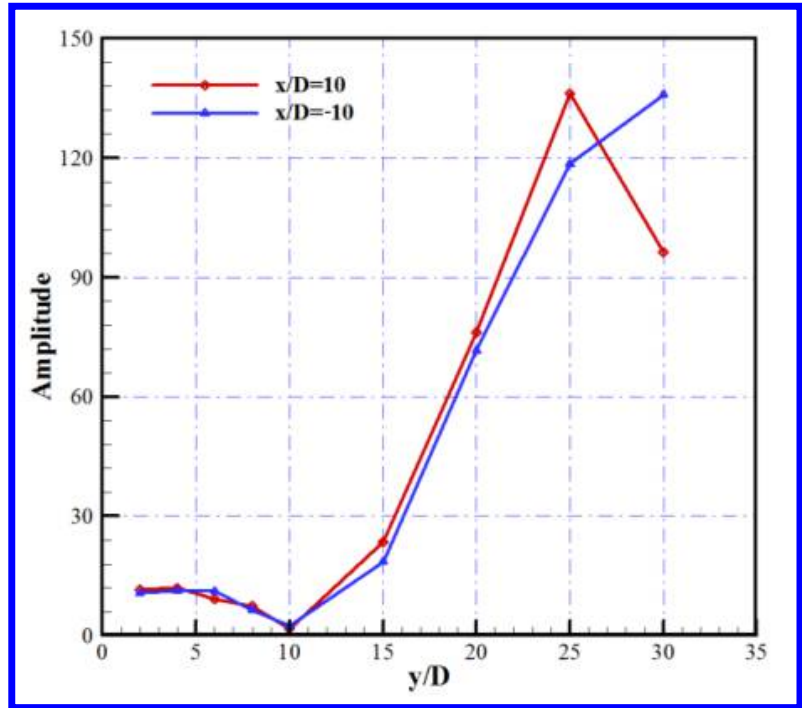

Figure 8. The amplitude of the screech tone at different locations on the streamwise lines

\section{Conclusion}

In this study, a LES modeling of a highly underexpanded jet with NPR=5.60 is conducted. The time-averaged flow fields are discussed and validated against previous experimental measurements and theoretical predictions. Especially, the height and diameter of Mach disk is in good agreement with that predicted previous studies. The 
dynamic process of sound source generation and convection is analyzed based on a series of snapshots of the instantaneous flow field, which shows the origination, emission, interaction and merging among sound sources as well as the reflection of sound wave against nozzle orifice. The $\mathrm{K}-\mathrm{H}$ wave and sound waves are well captured by the current LES modeling with high temporal resolution and high spatial resolution, then the dual-source pattern of the jet noise field is analyzed based on acoustic intensity distribution. Spectrums analysis of the pressure fluctuation at different locations are conducted to differentiate the directional K-H wave and circumferential sound wave generated by large-scale and small-scale turbulent structures.

\section{Acknowledgments}

The funding support for this work was provided by National Natural Science Foundation of China under contract 91016005 and LHD Youth Innovative Foundation under contract 0120481111. The authors are also grateful to National Supercomputer Center in Tianjin for providing computational resource.

\section{References}

${ }^{1}$ Heiser W H, Pratt D T. Hypersonic airbreathing propulsion [M]. AIAA, 1994.

${ }^{2}$ Raman G, Zaman K B M Q, Rice E J. Initial turbulence effect on jet evolution with and without tonal excitation [J]. Physics of Fluids A: Fluid Dynamics (1989-1993), 1989, 1(7): 1240-1248.

${ }^{3}$ Lighthill M J. On sound generated aerodynamically. I. General theory [J]. Proceedings of the Royal Society of London. Series A. Mathematical and Physical Sciences, 1952, 211(1107): 564-587.

${ }^{4}$ Raman G. Advances in understanding supersonic jet screech: review and perspective [J]. Progress in aerospace sciences, 1998, 34(1-2): 45-106.

${ }^{5}$ Umeda Y, Ishii R. On the sound sources of screech tones radiated from choked circular jets [J]. The Journal of the Acoustical Society of America, 2001, 110(4): 1845-1858.

${ }^{6}$ Liu J, Kailasanath K, Ramamurti R, et al. Large-eddy simulations of a supersonic jet and its near-field acoustic properties [J]. AIAA journal, 2009, 47(8): 1849-1865.

7 Tam C K W. Supersonic jet noise [J]. Annual Review of Fluid Mechanics, 1995, 27(1): 17-43.

${ }^{8}$ Lilley G M. On the noise from air jets [M]. Aeronautical Research Council, Noise Research Committee, 1948.

${ }^{9}$ Phillips O M. On the generation of sound by supersonic turbulent shear layers [J]. Journal of Fluid Mechanics, 1960, 9(01): $1-28$.

${ }^{10}$ Ribner H S. The generation of sound by turbulent jets [J]. Advances in Applied Mechanics, 1964, 8(103-182): 10.

$\checkmark{ }^{11}$ Crow S C, Champagne F H. Orderly structure in jet turbulence [J]. Journal of Fluid Mechanics, 1971, 48(03): 547-591.

$\checkmark 12$ Bradshaw P, Ferriss D H, Johnson R F. Turbulence in the noise-producing region of a circular jet [J]. Journal of Fluid Mechanics, 1964, 19(04): 591-624.

${ }^{13}$ Tam C K W, Viswanathan K, Ahuja K K, et al. The sources of jet noise: experimental evidence [J]. Journal of Fluid Mechanics, 2008, 615: 253-292.

${ }^{14}$ Panda J, Seasholtz R G. Measurement of shock structure and shock-vortex interaction in underexpanded jets using Rayleigh scattering [J]. Physics of Fluids (1994-present), 1999, 11(12): 3761-3777.

${ }^{15}$ Kolbe R L, Kailasanath K, Young T R, et al. Numerical studies of noise generation and mixing of supersonic rectangular jets[J]. High speed jet flows- 1995, 1995: 41-46.

${ }^{16}$ Kolbe R, Kailasanath K, Young T, et al. Numerical simulations of flow modification of supersonic rectangular jets [J]. AIAA journal, 1996, 34(5): 902-908.

${ }^{17} \mathrm{http}: / /$ www.openfoam.com/ 
${ }^{18}$ Chase, M.W. JANAF thermochemical tables. Journal of Physical and Chemical Reference Data, 1974, 3(2): 311 -480.

$\checkmark{ }^{19}$ A. Kurganov, E. Tadmor. New high-resolution central schemes for nonlinear conservation laws and convection-diffusion equations [J]. Journal of Computational Physics, 2001, 160: 241-282.

${ }^{20}$ Versteeg H K, Malalasekera W. An introduction to computational fluid dynamics: the finite volume method [M]. Pearson Education, 2007.

${ }^{21}$ Martin M P, Piomelli U, Candler G V. Subgrid-scale models for compressible large-eddy simulations [J]. Theoretical and Computational Fluid Dynamics, 2000, 13(5): 361-376.

${ }^{22}$ Fureby C, Möller S I. Large eddy simulation of reacting flows applied to bluff body stabilized flames [J]. AIAA journal, 1995, 33(12): 2339-2347.

${ }^{23}$ Tramecourt, N., Menon, S., and Amaya, J., LES of supercritical combustion in a gas turbine engine [C], in 40th AIAA/ASME/SAE/ASEE Joint Propulsion Conference and Exhibit, AIAA 2004-3381.

${ }^{24}$ Yoshizawa A, Horiuti K. Statistically derived subgrid scale kinetic energy model for Large-eddy simulation of turbulent flows [J]. Journal of the Physical Society of Japan, 1985, 54: 2834-2839.

${ }^{25}$ Gorle C, Gamba M, Ham F. Investigation of an underexpanded hydrogen jet in quiescent air using numerical simulations and experiments. Center for Turbulence Research Annual Research Briefs, Center for Turbulence Research, Stanford, CA.

${ }^{26}$ La Vuorinen V, Yu J, Tirunagari S. Large-eddy simulation of highly underexpanded transient gas jets [J]. Physics of Fluids, 2013, 25(1): 016101 .

$\checkmark 27$ A. Dauptain, B. Cuenot, Y. M. Gicquel. Large-eddy simulation of a stable supersonic jet impinging on flat plate [J]. AIAA, 2010, 48(10): 2325-2337.

${ }^{28}$ Z. A. Rana, B. Thornber, D. Drikakis. Transverse jet injection into a supersonic turbulent cross-flow [J]. Physics of Fluids, 2011, 23: 046103.

${ }^{29}$ YANG Meng. High speed pulsed schlieren technology and its application to flow visualization in supersonic combustion [D]. Master's Thesis. Institute of Mechanics, Chinese Academy of Science, 2012. (in Chinese)

${ }^{30}$ Ashkenas H, Sherman F. Structures and utilization of supersonic free jets in low density wind tunnels. NASA Technical Report 1965, No. CR-60423.

${ }^{31}$ B. C. R. Ewan and K. Moodie. Structures and velocity measurements in underexpanded jets [J]. Combustion Science and Technology, 1986, 45(5-6): 275-288.

${ }^{32}$ K.W. Tam, Directional acoustic radiation from a supersonic jet generated by shear layer instability [J].Journal of Fluid Mechanism, vol 46 757-768(1971).

${ }^{33}$ K.W. Tam, J.Panda et.al, The sources of jet noise: experimental evidence [J]. Journal of Fluid Mechanism,vol 615 253292(2008). 
This article has been cited by:

1. Wei Yao, weiyao@imech.ac.cn Chinese Academy of Sciences; Yueming Yuan, yuanym@imech.ac.cn Chinese Academy of Sciences; Xiaopeng Li, lixiaopeng@iet.cn Chinese Academy of Sciences; Jing Wang, wangjing@imech.ac.cn Chinese Academy of Sciences; Xuejun Fan, xfan@imech.ac.cn Chinese Academy of Sciences A comparative study of elliptical and round scramjet combustors by Improved Delayed Detached Eddy Simulation . [Citation] [PDF] [PDF Plus] 